\title{
Intoxicación por cloruro de magnesio: a propósito de un caso
}

\author{
Magnesium chloride poisoning: a case report
}

Joaquín Cordero-Villanuevaa ${ }^{1 *}$, Liliana Marín-Hinojosa ${ }^{1}$, Moisés Torres-Maure ${ }^{2,3}$ y Ximena Tapia-Ibáñez ${ }^{2}$

${ }^{1}$ Servicio de Emergencias, Hospital Nacional Dos de Mayo; ${ }^{2}$ Servicio de Emergencia, Centro de Información y Asistencia Toxicológica, Hospital Cayetano Heredia; ${ }^{3}$ Unidad de Información y Asistencia Toxicológica, Clínica San Pablo. Lima, Perú

\section{Introducción}

La hipermagnesemia es un trastorno muy raro y se define como una concentración de magnesio en sangre $>2.6 \mathrm{mg} / \mathrm{dl}(1.1 \mathrm{mmol} / \mathrm{l})$, aunque pudiera variar dependiendo del laboratorio, siendo el límite superior de normalidad $2 \mathrm{mg} / \mathrm{dl}^{1}$. Por lo general inician con sintomatología aquellos pacientes con concentraciones $>4.8 \mathrm{mg} / \mathrm{dl}(2 \mathrm{mmol} / \mathrm{l})$. Dentro de los mecanismos más descritos por los cuales los pacientes pueden presentar hipermagnesemia se encuentran la ingesta excesiva en forma de medicamentos, suplementos orales, rectales o por vía intravenosa, y la disminución en la excreción renal ya sea por lesión renal aguda o crónica, o una redistribución del espacio intracelular al extracelular y por liberación de células ${ }^{2,3}$.

\section{Presentación del caso}

Mujer de 42 años que ingresa al servicio de emergencias del Hospital Nacional Dos de Mayo, en Lima, Perú, tras iniciar en las 6 horas previas un cuadro caracterizado por palpitaciones, cefalea, náuseas, vómitos, sialorrea, visión borrosa, debilidad en las extremidades inferiores que impide la deambulación, dificultad para el habla, somnolencia y dificultad respiratoria. Este evento se desencadenó tras el consumo de aproximadamente $120 \mathrm{ml}$ de cloruro de magnesio (Fig. 1), el cual fue adquirido en una bodega naturista para el tratamiento sugerido de dolor de miembros inferiores. Sin antecedentes médicos ni quirúrgicos de importancia.

Al ingreso presenta las siguientes funciones vitales: presión arterial 140/100 mmHg, frecuencia cardiaca 94 latidos por minuto, frecuencia respiratoria 23 respiraciones por minuto, temperatura $37^{\circ} \mathrm{C}$, saturación de oxígeno 95\% y glucometría capilar 169 mg/dl. La exploración física revela escala de coma de Glasgow 13 puntos, somnolienta, orientada en las tres esferas, pupilas isocóricas fotorreactivas, flacidez de las cuatro extremidades, reflejos osteotendinosos disminuidos, a nivel respiratorio amplexación bilateral disminuida, sin ruidos agregados, precordio sin presencia de soplos audibles, aspecto marmóreo en abdomen y muslos, peristalsis presente, sin datos de irritación peritoneal, y extremidades con presencia de edema con fóvea $(+/+++)$ bimaleolar.

\section{Abordaje}

Al ingreso se realizan los exámenes séricos, que muestran los siguientes valores alterados: leucocitosis sin desviación izquierda 25,050 $\mathrm{mm}^{3}$, abastonados $1 \%$, plaquetas $287,000 / \mathrm{mm}^{3}$, hemoglobina $12.6 \mathrm{~g} / \mathrm{dl}$, hematocrito $41.2 \%$, velocidad corpuscular media 93.8 , hemoglobina corpuscular media 28.7, segmentados

\section{Correspondencia:}

*Joaquín Cordero-Villanueva

E-mail: jocorderovi@gmail.com
Fecha de recepción: 30-10-2021

Fecha de aceptación: 17-12-2021 DOI: 10.24875/REIE.21000135
Disponible en internet: 07-03-2022

Rev Educ Investig Emer. 2022;4(Supl 1):86-89 www.medicinadeemergencias.com bajo la licencia CC BY-NC-ND (http://creativecommons.org/licenses/by-nc-nd/4.0/) 


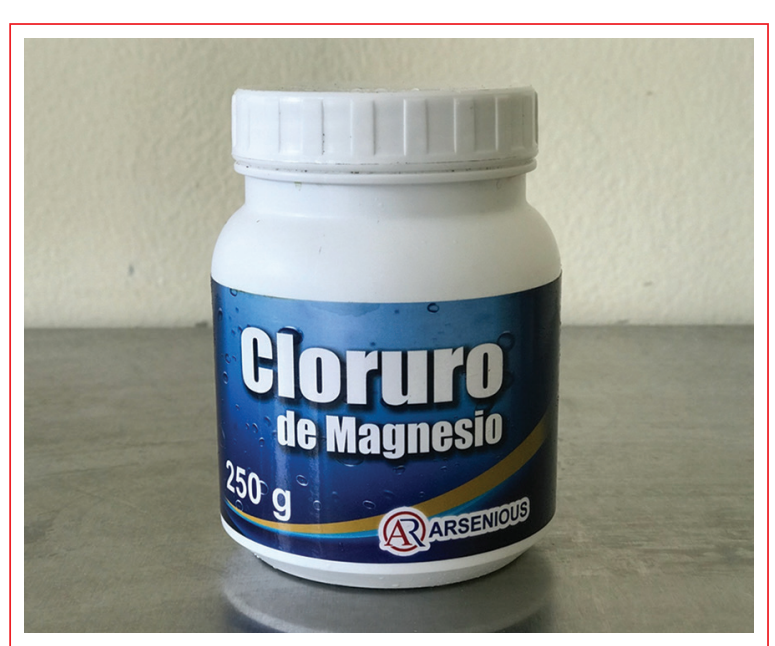

Figura 1. Frasco de $250 \mathrm{~g}$ de cloruro de magnesio hexahidratado $\left(\mathrm{MgCl}_{2} \cdot 6 \mathrm{H}_{2} \mathrm{O}\right)$.

$88.2 \%$, tiempo de protrombina $14.10 \mathrm{~s}$, International Normalized Ratio 1.04, tiempo parcial de tromboplastina activada $23.5 \mathrm{~s}$, fibrinógeno $576 \mathrm{mg} / \mathrm{dll}$, magnesio $15.48 \mathrm{mg} / \mathrm{dl}$, cloro $121.34 \mathrm{mmol} / \mathrm{l}$, potasio $4.06 \mathrm{mmol} / \mathrm{l}$, sodio $140.22 \mathrm{mmol} / \mathrm{l}$, fósforo $6.36 \mathrm{mg} / \mathrm{dl}$, calcio $8.99 \mathrm{mg} / \mathrm{dl}$, gasometría arterial con acidosis metabólica hiperclorémica-hiperlactatémica junto con acidosis respiratoria ( $\mathrm{pH} 7.1, \mathrm{HCO}_{3} 11.3, \mathrm{pCO}_{2} 31.5$, lactato 4. 1) y con hipoxemia $\left(\mathrm{pO}_{2} 62.3\right)$ (Tabla 1). Examen de orina: $\mathrm{pH} 5.0$, proteinuria (+++), glucosa (+), células epiteliales 1 , leucocitos 0 , hematíes 4 , glucosa $183.57 \mathrm{mg} / \mathrm{dl}$, proteína $C$ reactiva $<5 \mathrm{mg} / \mathrm{l}$. Se realiza electrocardiograma (Fig. 2), en el que se aprecia un infradesnivel de PR en las derivadas DII y AVF, $V_{2}$ y $V_{3}$ y QTc en 440 ms.

Durante su estancia se realizan gasometrías seriadas de control, reportando a las 12 horas $\mathrm{pH} 7.167$, $\mathrm{pCO}_{2} 39.3, \mathrm{HCO}_{3} 13.6$ y lactato 3.7 ), y a las 24 horas $\mathrm{pH}$ 7.38, $\mathrm{pCO}_{2} 33.5, \mathrm{pO}_{2} 64.6, \mathrm{HCO}_{3} 19.4$ y lactato 1.4. Se determinan nuevamente los valores de magnesio, que son de $5.39 \mathrm{mg} / \mathrm{dl}$.

\section{Tratamiento}

Se brinda manejo con agua destilada en infusión, furosemida (40 mg en bolo y luego $20 \mathrm{mg} / 8 \mathrm{~h}$ ), gluconato de calcio $10 \%$ cada 8 horas, bicarbonato de sodio $8.4 \%$ (cuatro ampollas en bolo y luego dos ampollas cada 8 horas). Por los valores de magnesio registrados y por encontrarse con datos de intoxicación se inicia terapia de reemplazo renal, con un total de dos sesiones en 24 horas.
Tabla 1. Valores gasométricos de ingreso y control a las 12 horas y 24 horas; niveles de magnesio en sangre a su ingreso y a las 24 horas post tratamiento

\begin{tabular}{|l|c|c|c|}
\hline & Ingreso & $\mathbf{1 2} \mathbf{h}$ & $\mathbf{2 4} \mathbf{~ h}$ \\
\hline $\mathrm{pH}$ & 7.1 & 7.1 & 7.3 \\
\hline $\mathrm{CO}_{2}$ & 31.5 & 39.3 & 33.5 \\
\hline $\mathrm{HCO}_{3}$ & 11.3 & 13.6 & 19.4 \\
\hline Lactato & 4.1 & 3.7 & 1.4 \\
\hline Magnesio & 15.48 & & 5.39 \\
\hline
\end{tabular}

\section{Desenlace y seguimiento}

Paciente que ingresa al servicio de urgencias y se inicia hidratación intensa y estimulación de la diuresis. Al contar con los resultados de los exámenes séricos se corrobora una hipermagnesemia grave sintomática (15.5 mg/dl), motivo por el cual se inicia terapia dialítica de urgencia, dos sesiones en 24 horas, en las cuales la paciente permanece hemodinámicamente estable y sin necesidad de manejo ventilatorio invasivo. Se aprecia mejoría clínica y electrocardiográfica (Fig. 3), que se correlaciona con la disminución del valor sérico de magnesio $(5.39 \mathrm{mg} / \mathrm{dl})$ a las 24 horas.

\section{Discusión}

El magnesio es el segundo catión intracelular más abundante en el cuerpo humano, encontrándose aproximadamente un 50-60\% en el hueso como sustituyente superficial del componente mineral hidroxiapatita ${ }^{2,4}$, mientras que el $31 \%$ está en el espacio intracelular y el $2 \%$ en el espacio extracelular ${ }^{5}$. Siendo este electrolito un cofactor en más de 300 reacciones enzimáticas, ayuda a estabilizar las enzimas y las reacciones generadoras de adenosín trifosfato, el cual es requerido para múltiples procesos, como la síntesis de grasa, la utilización de la glucosa, la síntesis de proteínas y ácidos nucleicos, y la contracción y la relajación muscular, y además regula el tono vascular y el ritmo cardiaco ${ }^{6}$. Dentro de las manifestaciones clínicas más frecuentes se encuentran síntomas neuromusculares, parestesia facial, debilidad muscular progresiva a parálisis flácida, depresión respiratoria y apnea; en hipermagnesemias graves pueden encontrarse hipotensión, bradicardia, bloqueo cardiaco completo y paro cardiaco; también se han descrito náuseas y vómitos ${ }^{1,7-9}$.

Dentro del tratamiento se incluyen rehidratación basada en parámetros dinámicos, calcio para mejorar la disfunción cardiaca, soporte cardiorrespiratorio si es 


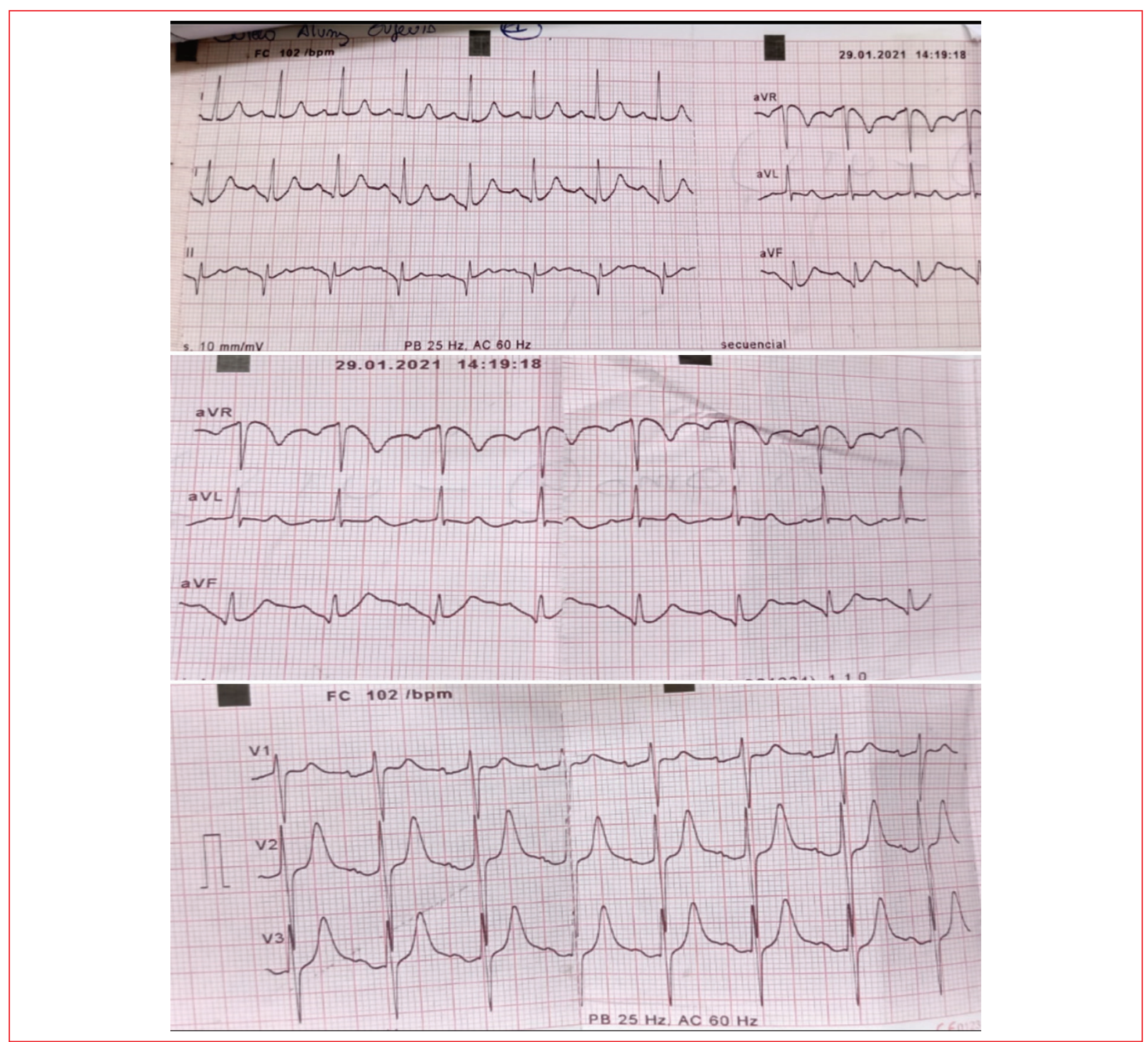

Figura 2. Electrocardiograma con evidencia desnivel negativo del segmento PR de forma difusa principalmente en derivaciones $\mathrm{V}_{1}, \mathrm{~V}_{2}, \mathrm{~V}_{3}$, ondas $\mathrm{T}$ acuminadas y barras asimétricas $\left(\mathrm{V}_{2} \mathrm{y} \mathrm{V}_{3}\right)$, intervalo $\mathrm{OT}$ corregido por Hodges $465 \mathrm{~ms}$.

preciso, uso de diuréticos, considerar terapia de reemplazo renal para una mayor eliminación, con monitoreo constante de signos vitales y reposición en caso necesario del resto de electrolitos (Yamaguchi et al., 2019). Realizando este manejo, nuestra paciente mejoró significativamente en un periodo de 24 horas, sin necesidad de soporte ventilatorio invasivo y con reversión de los cambios electrocardiográficos. Podemos considerar que nuestra paciente contó con hipermagnesemia sintomática, con valores tan altos que podrían ser explicados si de manera concomitante tuviera una lesión renal aguda o crónica, presentando proteinuria 3+, lo cual podría indicar la presencia de alguna nefropatía, junto con la ingesta concomitante de dosis muy altas de cloruro de magnesio.

\section{Perspectivas del paciente}

El tratamiento multidisciplinario de los pacientes intoxicados se debe considerar para cualquier tipo de xenobiótico o veneno al que haya podido estar expuesto el paciente; esto asegurará que el manejo se realice de manera oportuna y eficaz, tomando decisiones terapéuticas que tendrán un impacto a corto, mediano y largo plazo en el pronóstico de los pacientes.

El abordaje que se brindó a esta paciente desde el servicio de urgencias y su manejo, así como la 


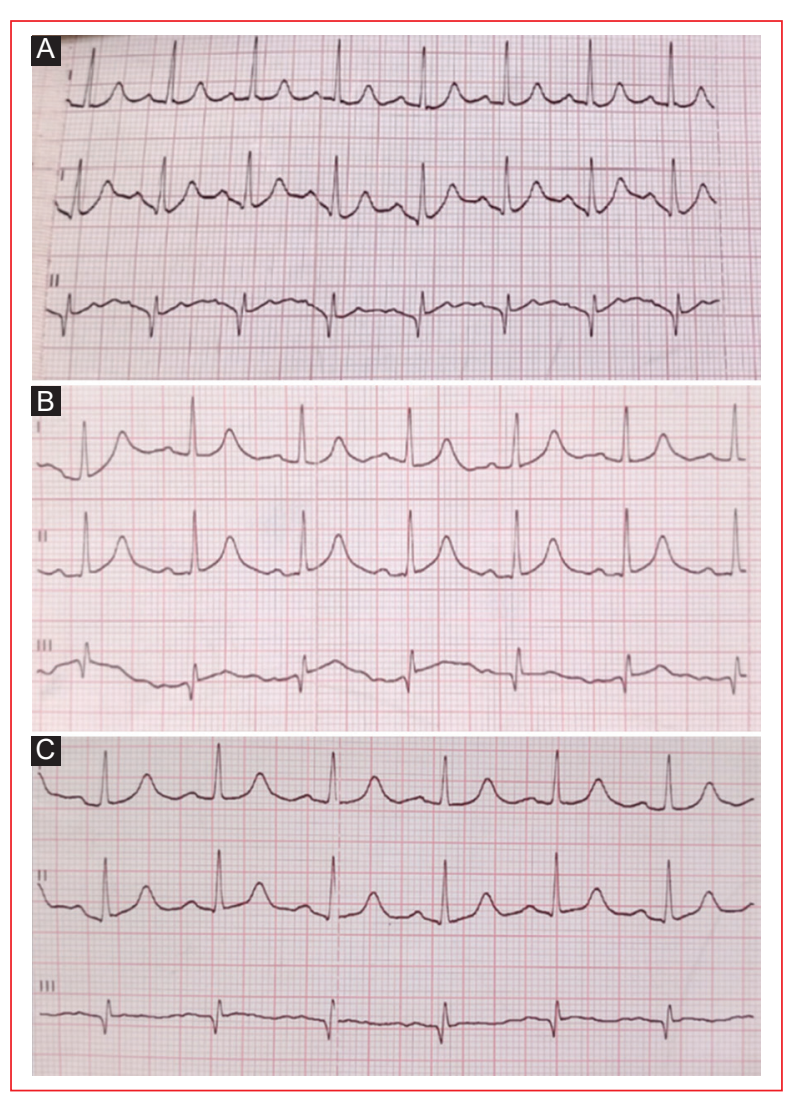

Figura 3. Electrocardiogramas, disminución progresiva del QT corregido. A: electrocardiograma 1: QTc en 440 ms. B: electrocardiograma 2: 0Tc en $436 \mathrm{~ms}$. C: electrocardiograma 3: 0Tc en $423 \mathrm{~ms}$.

interconsulta de manera oportuna a la especialidad de toxicología clínica para decidir si era candidata a algún método de eliminación específico, y por ende la intervención de parte de nefrología para la realización de la terapia de reemplazo renal, fueron determinantes ante los elevados valores de magnesio y la sintomatología que presentaba.

\section{Agradecimientos}

A los profesionales de salud que fallecieron durante la lucha contra la pandemia de COVID-19.

\section{Financiamiento}

Este trabajo fue autofinanciado.

\section{Conflicto de intereses}

Para este trabajo no existen conflictos de intereses.

\section{Responsabilidades éticas}

Protección de personas y animales. Los autores declaran que para esta investigación no se han realizado experimentos en seres humanos ni en animales.

Confidencialidad de los datos. Los autores declaran que han seguido los protocolos de su centro de trabajo sobre la publicación de datos de pacientes.

Derecho a la privacidad y consentimiento informado. Los autores han obtenido el consentimiento informado de los pacientes y/o sujetos referidos en el artículo. Este documento obra en poder del autor de correspondencia.

\section{Bibliografía}

1. Chang WTW, Radin B, McCurdy MT. Calcium, magnesium, and phosphate abnormalities in the emergency department. Emerg Med Clin North Am. 2014;32:349-66.

2. Jahnen-Dechent W, Ketteler M. Magnesium basics. Clin Kidney J. 2012;5(Suppl 1):i3-i14

3. Kala J, Abudayyeh A. Magnesium: an overlooked electrolyte. J Emerg Med. 2017;52:741-3.

4. Karahan MA, Kucuk A, Buyukfirat E, Yalcin F. Acute respiratory and renal failure due to hypermagnesemia, induced by counter laxatives in an elderly man. J Clin Diagn Res. 2015;9:UL01.

5. Moe SM. Disorders involving calcium, phosphorus, and magnesium. Prim Care. 2008;35:215-37.

6. Saris NE, Mervaala E, Karppanen H, Khawaja JA, Lewenstam A. Magnesium. An update on physiological, clinical and analytical aspects. Clin Chim Acta. 2000;294:1-26.

7. Sugiyama M, Kusumoto E, Ota M, Kimura Y, Tsutsumi N, Oki E, et al. Induction of potentially lethal hypermagnesemia, ischemic colitis, and toxic megacolon by a preoperative mechanical bowel preparation: report of a case. Surg Case Rep. 2016;2:18.

8. Tatsumi $H$, Masuda $Y$, Imaizumi $H$, Kuroda $H$, Yoshida S, Kyan R, et al. A case of cardiopulmonary arrest caused by laxatives-induced hypermagnesemia in a patient with anorexia nervosa and chronic renal failure. J Anesth. 2011;25:935-8.

9. Whang R. Clinical disorders of magnesium metabolism. Compr Ther. 1997;23:168-73.

10. Yamaguchi H, Shimada H, Yoshita K, Tsubata Y, Ikarashi K, Morioka T, et al. Severe hypermagnesemia induced by magnesium oxide ingestion: a case series. CEN Case Rep. 2019;8:31-7. 